\title{
Diffuse interstellar bands in fullerene planetary nebulae: the fullerenes - diffuse interstellar bands connection ${ }^{\star}$
}

\author{
D. A. García-Hernández ${ }^{1,2}$ and J. J. Díaz-Luis ${ }^{1,2}$ \\ 1 Instituto de Astrofísica de Canarias, C/ via Láctea s/n, 38200 La Laguna, Spain \\ e-mail: agarcia@iac.es \\ 2 Departamento de Astrofísica, Universidad de La Laguna (ULL), 38206 La Laguna, Spain \\ Received 14 December 2012 / Accepted 1 January 2013
}

\section{ABSTRACT}

\begin{abstract}
We present high-resolution $(R \sim 15000)$ VLT/UVES optical spectra of two planetary nebulae (PNe; Tc 1 and M 1-20) where $\mathrm{C}_{60}$ (and $\left.\mathrm{C}_{70}\right)$ fullerenes have already been found. These spectra are of high quality $(S / N>300)$ for PN Tc 1 , which permits us to search for the expected electronic transitions of neutral $\mathrm{C}_{60}$ and diffuse interstellar bands (DIBs). Surprisingly, we report the non-detection of the most intense optical bands of $\mathrm{C}_{60}$ in $\mathrm{Tc} 1$, although this could be explained by the low $\mathrm{C}_{60}$ column density estimated from the $\mathrm{C}_{60}$ infrared bands if the $\mathrm{C}_{60}$ emission peaks far away from the central star. The strongest and most common DIBs in both fullerene PNe are normal for their reddening. Interestingly, the very broad $4428 \AA$ DIB and the weaker $6309 \AA$ DIB are found to be unusually intense in Tc 1 . We also report the detection of a new broad $(F W H M \sim 5 \AA$ ) unidentified band at $\sim 6525 \AA$. We propose that the $4428 \AA$ DIB (probably also the $6309 \AA$ DIB and the new $6525 \AA$ band) may be related to the presence of larger fullerenes (e.g., $\mathrm{C}_{80}$, $\mathrm{C}_{240}, \mathrm{C}_{320}$, and $\mathrm{C}_{540}$ ) and buckyonions (multishell fullerenes such as $\mathrm{C}_{60} @ \mathrm{C}_{240}$ and $\mathrm{C}_{60} @ \mathrm{C}_{240} @ \mathrm{C}_{540}$ ) in the circumstellar envelope of Tc 1 .
\end{abstract}

Key words. astrochemistry - line: identification - circumstellar matter - ISM: molecules - planetary nebulae: individual: Tc 1 planetary nebulae: individual: M 1-20

\section{Introduction}

The diffuse interstellar bands (DIBs) have remained a mystery for astronomers since their discovery about ninety years ago (Heger 1922); they are one of the long-standing problems in the interstellar medium (ISM). Nowadays, more than 400 DIBs have been identified in the ISM (e.g., Hobbs et al. 2008; Geballe et al. 2011). No DIB carrier has been convincingly identified, although more recent studies suggest that the DIB carriers may be complex molecules containing carbon (see e.g., Herbig 1995; Snow \& McCall 2006; Cox 2011). Polycyclic aromatic hydrocarbons (PAHs; e.g., Salama et al. 1999), fullerenes (e.g., Foing \& Ehrenfreund 1994; Herbig 2000; Iglesias-Groth 2007), and polyatomic organic molecules (e.g., Maier et al. 2011) are among the proposed DIB carriers. In particular, the fullerenes - DIB hypothesis may also explain the intense UV absorption band at $217 \mathrm{~nm}$ as due to fullerene-based molecules such as buckyonions (multishell fullerenes; e.g., Iglesias-Groth 2004) and hydrogenated fullerenes (e.g., Cataldo \& Iglesias-Groth 2009).

The 9577 and $9632 \AA$ DIBs observed in a few hot reddened stars lie near two electronic transitions of the $\mathrm{C}_{60}$ cation observed in rare gas matrices (Foing \& Ehrenfreund 1994). However, the presence of fullerenes in astrophysical environments has been a matter of debate until recently when Spitzer observations have provided evidence for $\mathrm{C}_{60}$ and $\mathrm{C}_{70}$ fullerenes from planetary nebulae (PNe; Cami et al. 2010; García-Hernández et al. 2010, 2011a, 2012a), reflection nebulae (Sellgren et al. 2010) and the least H-deficient R Coronae Borealis (RCB)

\footnotetext{
* Appendix $\mathrm{A}$ is available in electronic form at http://www . aanda.org
}

stars (García-Hernández et al. 2011b,c). None of these environments is highly hydrogen-deficient. Furthermore, the recent detection of $\mathrm{C}_{60}$ fullerenes in PNe with normal $\mathrm{H}$-abundances (García-Hernández et al. 2010, 2011a, 2012a) suggests that large fullerenes may be formed as decomposition products of hydrogenated amorphous carbon (HAC) dust and that fullerenes may be not so exotic and can form under conditions that are common to essentially all solar-like stars at the end of their lives.

Thus, fullerenes and related large carbon-based species (e.g., other fullerenes as stable exohedral or endohedral metallocomplexes; Kroto \& Jura 1992) might be ubiquitous in the ISM and continue to be serious candidates for DIB carriers (e.g., Bettens \& Herbst 1996; Herbig 1995; Iglesias-Groth 2007). However, a detailed analysis of the DIBs towards fullerenecontaining - accompanied or not by PAH molecules (e.g., García-Hernández et al. 2012b) - astrophysical environments is mandatory before one can reach conclusions about the nature of the DIB carriers. In this context, the recent infrared detection of large fullerenes in PNe offers the beautiful opportunity for studying the DIB spectrum of sources where fullerenes are abundant. In this Letter we present for the first time a detailed inspection of the optical spectra of two fullerene PNe, which permits us to directly explore the fullerenes - DIB connection.

\section{Optical VLT/UVES spectroscopy of fullerene PNe}

We acquired optical spectra of the fullerene PNe Tc 1 ( $B=11.1$, $E(B-V)=0.23$; Williams et al. 2008) and M 1-20 ( $B=13.7$, $E(B-V)=0.80$; Wang \& Liu 2007). Tc 1 displays a fullerenedominated spectrum with no clear signs of PAHs while M 1-20 also shows PAH features (García-Hernández et al. 2010). The 
observations were carried out at the ESO VLT (Paranal, Chile) in service mode between May and September 2011. The optical spectra were taken in the wavelength ranges $\sim 3300-4500$, 5750-7500, and 7700-9400 ̊ with UVES at the UT2 telescope using the 2.4" slit with the standard setting DIC2 $(390+760)$. This configuration gives a resolving power of $\sim 15000$ and an adequate interorder separation. We required $R \sim 15000$ to appropriately sample the relatively broad $\mathrm{C}_{60}$ features together with the generally narrower DIBs (e.g., 5797, 5850, 6196, 6614 $\AA$ ). To detect weak broad $(\geq 4 \AA) \mathrm{C}_{60}$ features in the optical we aimed for a minimum signal-to-noise ratio (S/N) of 200 around $3760 \AA$.

As comparison stars for Tc 1 we selected the nearby B-type star HR $6334(B=5.1 ; E(B-V)=0.42$; Wegner 2003) and for M 1-20 HR $6716(B=5.7 ; E(B-V)=0.22$; Wegner 2003). Both comparison stars were observed on the same dates as the corresponding PNe using the same VLT/UVES set-up. The observed spectra were processed by the UVES data reduction pipeline (Ballester et al. 2000) and the stellar continuum was fitted by using standard astronomical tasks in IRAF.

For Tc 1, we obtained 10-12 individual exposures (of $900 \mathrm{~s}$ each) in the $~ 3300-4500,5750-7500$, and 7700-9400 $\AA$ spectral ranges, giving total exposure times of $2.5-3 \mathrm{~h}$. The $\mathrm{S} / \mathrm{N}$ in the continuum in the summed Tc 1 spectrum is $\sim 300$ at $4000 \AA$ and higher than 350 at wavelengths longer than $6000 \AA$. For the fainter PN M 1-20, however, we only could obtain eight individual exposures of $1800 \mathrm{~s}$ each in both $\sim 5750-7500$ and 7700-9400 ̊ spectral regions, giving a total exposure time of four hours. The $\mathrm{S} / \mathrm{N}$ in the individual $1800 \mathrm{~s}$ exposures in the blue region (3300-4500 $\AA$ ) was too low $(<10)$. During the execution of our service program we decided to use a binning $2 \times 2$ to increase the $\mathrm{S} / \mathrm{N}$ around $4000 \AA$ but we only obtained two individual exposures of $1800 \mathrm{~s}$ each. Thus, the $\mathrm{S} / \mathrm{N}$ in the continuum in the best M 1-20 spectra is $\sim 20$ at $4000 \AA$ and higher than 30 at wavelengths longer than $6000 \AA$. Finally, an S/N in excess of 450 was easily achieved in the summed spectra of the bright comparison stars HR 6334 and HR 6716 by using total exposure times of several minutes.

Unfortunately, the $\mathrm{S} / \mathrm{N}$ in the $\mathrm{M} 1-20$ spectrum is too low to search for weak and broad absorption bands (e.g., neutral $\mathrm{C}_{60}$ features, see below) in its spectrum but it was found to be enough to detect and characterize several of the strongest and most common DIBs towards this PN with a rather high $E(B-V)$ of 0.80 .

\section{Electronic transitions of neutral $\mathrm{C}_{60}$}

We have searched the high-quality $(S / N>300)$ spectra of the PN Tc 1 (see above) for electronic transitions of $\mathrm{C}_{60}{ }^{1}$. The strongest electronically allowed transitions of neutral gasphase $\mathrm{C}_{60}$ molecules, as measured in the laboratory, are located around $\sim 3760,3980$, and $4024 \AA$ with widths of 8,6 , and $4 \AA$ (Sassara et al. 2001; see also García-Hernández et al. 2012b). The strongest $\mathrm{C}_{60}$ transition seen in the laboratory spectra is that at $3980 \AA$ (Sassara et al. 2001).

Surprisingly, we can find no evidence for the presence of neutral $\mathrm{C}_{60}$ in absorption (or emission) at the wavelengths of the expected electronic transitions mentioned above. This is shown in Fig. A.1 (in the Appendix) where we compare the Tc 1

\footnotetext{
$\mathrm{C}_{70}$ is ten times less abundant than $\mathrm{C}_{60}$ (e.g., García-Hernández et al. 2012a) and we do not find evidence for extra absorption around ( $\pm 10 \AA)$ the expected $\mathrm{C}_{70}$ electronic transitions (e.g., Ajie et al. 1990).
}

velocity-corrected spectra ${ }^{2}$ with those of the nearby B-type star HR 6334 around the expected positions of the strongest $\mathrm{C}_{60}$ transitions. It is to be noted here that the 3760 and $4024 \AA$ bands coincide with several strong O lines and He I $4026 \AA$ line, respectively, which hampers the identification of broad and weak absorption features. However, the spectral region around $3980 \AA$ (Fig. A.1 in the Appendix, right panel) is free from any contaminant and there is no evidence for the presence of the neutral $\mathrm{C}_{60}$ feature at this wavelength.

The one-sigma detection limits on the equivalent widths (EQWs) derived from our Tc 1 spectra are 63, 20, and $12 \mathrm{~m} \AA$ for the 3760,3980 , and $4024 \AA \mathrm{C}_{60}$ transitions ${ }^{3}$. This translates into column densities of $6 \times 10^{12}, 1 \times 10^{13}$, and $6 \times 10^{12} \mathrm{~cm}^{-2}$. We can compare this column density limit of about $10^{13} \mathrm{~cm}^{-2}$ with estimates of the circumstellar density of $\mathrm{C}_{60}$ molecules, taking into account the total number of $\mathrm{C}_{60}$ molecules $\left(N\left(\mathrm{C}_{60}\right)=1.8 \times 10^{47}\right.$ for $d=2 \mathrm{kpc}$; García-Hernández et al. 2011a) calculated from the IR $\mathrm{C}_{60}$ features. By following García-Hernández et al. (2012b, Eqs. (1)-(3)), we can estimate the density of $\mathrm{C}_{60}$ molecules by assuming a spherical shell of radius $\left(R_{\text {out }}-R_{\text {in }}\right)$ and a uniform number of $\mathrm{C}_{60}$ molecules throughout the shell. Thus, considering that $L=1480 L_{\odot}$ for Tc 1 (Pottasch et al. 2011), we obtain dust temperatures $\left(T_{\mathrm{d}}\right)$ of $415 \mathrm{~K}$ at $\sim 18$ au and $100 \mathrm{~K}$ at $301 \mathrm{au}$, where the first temperature is the $\mathrm{C}_{60}$ excitation temperature (García-Hernández et al. 2011a) and the latter temperature corresponds to the minimum temperature of the dust to be detected in the mid-IR by Spitzer. For a distance of $2 \mathrm{kpc}$, we estimate a circumstellar density of fullerenes $n\left(\mathrm{C}_{60}\right)=0.46 \mathrm{~cm}^{-3}$ and a $\mathrm{C}_{60}$ column density along the path $\left(R_{\text {out }}-R_{\text {in }}\right)$ of $\sim 2 \times 10^{15} \mathrm{~cm}^{-2}$; a lower $\mathrm{C}_{60}$ column density of $3 \times 10^{14} \mathrm{~cm}^{-2}$ is obtained for $L=10^{4} L_{\odot}$. Thus, our estimate of the $\mathrm{C}_{60}$ column density is 200-300 times higher than the observed upper limits. It is to be noted here that although the Spitzer/IRS observations contain a marginal amount of spatial information $\left(\sim 2^{\prime \prime} / \mathrm{pixel}\right)$ and that mid-IR images at much higher spatial resolution would be desirable, Bernard-Salas et al. (2012) presented tentative evidence that the $8.5 \mu \mathrm{m}$ emission (and attributed to $\mathrm{C}_{60}$ ) in Tc 1 is extended and peaks at 2-3 pixels ( $\sim 6400-9700$ au for $d=2 \mathrm{kpc})$ from the central star. By assuming that fullerenes are uniformly distributed in a shell of $R_{\text {out }}=9700$ au and $R_{\text {in }}=6400 \mathrm{au}$, the estimated $\mathrm{C}_{60}$ column density $\left(\sim 1 \times 10^{12} \mathrm{~cm}^{-2}\right)$ is a factor between 6 and 10 below our observed upper limits.

In short, our $\mathrm{C}_{60}$ column density values estimated from the $\mathrm{C}_{60}$ IR bands could explain the non-detection of the electronic $\mathrm{C}_{60}$ transitions in our Tc 1's optical spectra only if the $\mathrm{C}_{60}$ emission peaks far away from the central star. However, we cannot discard that the line of sight to Tc 1 may not intersect the fullerene-rich regions of the circumstellar shell (e.g., if the fullerenes may be formed in clumps). On the other hand, one can speculate that the strongest $3980 \AA \mathrm{C}_{60}$ band - perhaps the other electronic bands, too - could be suppresed if the fullerenes are in the solid-state phase (e.g., Evans et al. 2012; García-Hernández et al. 2012a). However, a laboratory spectrum of $\mathrm{C}_{60}$ dissolved in $n$-hexane displays the same transitions seen in the gas-phase (Cataldo, priv. comm.). An alternative and more

\footnotetext{
2 We applied average velocity corrections of $-105.8 \mathrm{~km} \mathrm{~s}^{-1}(\sim-1.4 \AA)$ for Tc 1 and of $20.1 \mathrm{~km} \mathrm{~s}^{-1}$ ( $\sim 0.3 \AA$ ) for HR 6334 .

3 One-sigma detection limits for the EQWs in our spectra scale as $\sim 1.064 \times F W H M /(S / N)$ (see e.g., Hobbs et al. 2008) but this value for the $3760 \AA$ band is estimated by modeling and subtracting the oxygen lines around $3760 \AA$.
} 

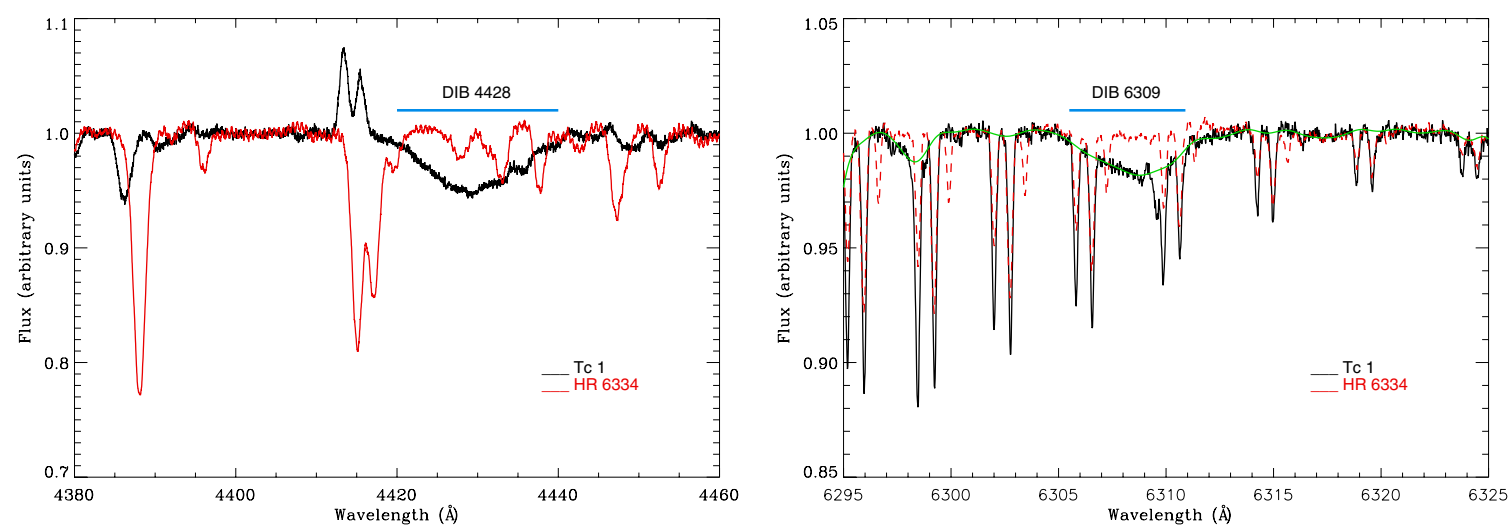

Fig. 1. Spectra of Tc 1 (in black) and HR 6334 (in red) around the $4428 \AA$ (left panel) and $6309 \AA$ A DIBs (right panel). Both DIBs are found to be unusually strong in Tc 1 while HR 6334 - with a higher $E(B-V)$ of 0.42 - does not show evidence of their presence. The telluric line corrected spectrum of Tc 1 around $6309 \AA$ (right panel) is shown by the smooth line (in green).

exotic explanation may be that the mid-IR features seen in Tc 1 are not due to $\mathrm{C}_{60}$ and $\mathrm{C}_{70}$ solely, being contaminated by other more complex fullerene-based molecules. This latter interpretation seems to be supported by our study of the DIBs towards Tc 1 and presented below.

\section{Diffuse interstellar bands in fullerene PNe}

We have followed the catalog of DIBs measured in the high-S/N HD 204287's spectrum (Hobbs et al. 2008) to search them in the VLT/UVES spectrum of Tc 1 and M 1-20. However, we concentrate here on analyzing eight of the strongest DIBs most commonly found in the ISM as well as on detecting unusually strong DIBs (i.e., not present in the nearby comparison stars and/or in Hobbs et al. 2008), which could be potentially due to fullerenes or fullerene-based molecules. Thus, we can compare the characteristic of most common DIBs for both PNe in our sample as well as with existing literature data on field-reddened stars (e.g., Luna et al. 2008). The exhaustive analysis of the weaker DIBs listed by Hobbs et al. (2008) and also detected in Tc 1's spectrum will be published elsewhere.

Our list of DIBs in Tc 1 and M 1-20 are listed in Table A.1 (in the Appendix), where we give the measured central wavelength, FWHM, EQW, the $\mathrm{S} / \mathrm{N}$ in the neighboring continuum, and the normalized equivalent widths $\mathrm{EQW} / E(B-V)$. For comparison we also list the EQW/E $(B-V)$ measured in HD 204827 and field-reddened stars by Hobbs et al. (2008) and Luna et al. (2008). It should be noted that we could not estimate the total absorption of the 6993 and $7223 \AA$ DIBs (not shown in Table A.1 in the Appendix) in our PNe because of the strong meddling from the telluric lines. For the well-studied DIBs at 5780, 5797, 5850, 6196, 6270, 6284, 6380, and $6614 \AA^{4}$, the EQW/E $(B-V)$ in fullerene $\mathrm{PNe}$ agree reasonably well with the values reported in HD 204827 (Hobbs et al. 2008) and field-reddened stars (Luna et al. 2008). The observed nearby comparison stars HR 6334 and HR 6716 also display EQW/E $(B-V)$ values completely consistent (within the errors) with those measured in our PNe. This indicates that the carriers of the latter well-studied DIBs are not particularly overabundant in fullerene PNe.

Interestingly, the well-studied DIB at $4428 \AA$ as well as the weaker $6309 \AA$ DIB listed by Hobbs et al. (2008) are found to be unusually strong towards Tc 1 . These last DIBs are not detected

\footnotetext{
4 Note that a possible exception is the $6284 \AA$ DIB but its strength is known to be not very well correlated with the interstellar reddening (e.g., Luna et al. 2008).
}

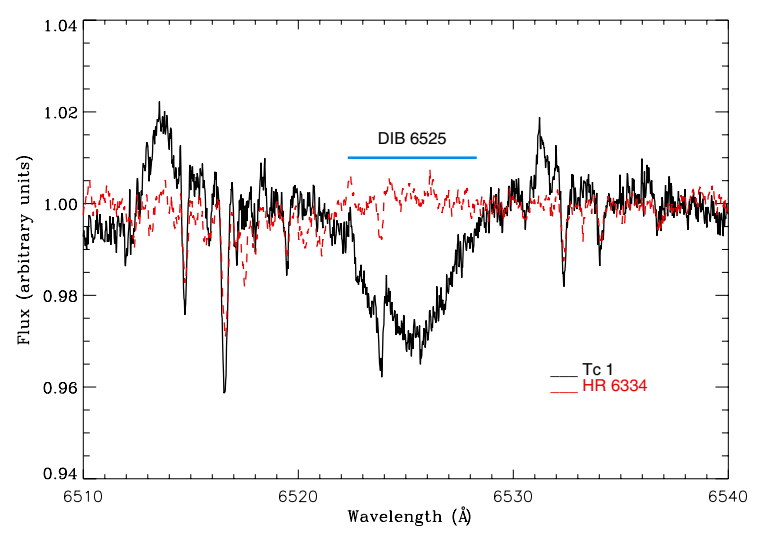

Fig. 2. Spectral region around the new broad unidentified band at $6525 \AA$ A in Tc 1 (in black) and HR 6334 (in red).

in the nearby comparison star HR 6334 with a higher $E(B-V)$ of 0.42 . Figure 1 compares the 4428 and $6309 \AA$ DIBs in Tc 1 with those in HR 6334. Adopting a Lorentzian profile for the $4428 \AA$ DIB (Snow et al. 2002), we obtain EQW $=860 \mathrm{~m} \AA$, which is at least a factor of two greater than expected for the low reddening of $E(B-V)=0.23$ in Tc 1; see e.g., Figs. 6 and 15 in Snow et al. (2002) and van Loon et al. (2012). We note that there is tentative evidence for an unusually strong (5-10\% of the continuum and $E Q W=2579 \pm 786 \mathrm{~m} \AA$ ) $4428 \AA \mathrm{DIB}$ in M 1-20, too (see Fig. A.2 in the Appendix), something that supports our finding in Tc 1 . However, we prefer to be cautious until higher S/N M 1-20 spectra are obtained. On the other hand, the uncommon $6309 \AA$ DIB in Tc 1 appears to be three times more intense than that observed in HD 204287 by Hobbs et al. (2008, see Table A.1 in the Appendix). Finally, an unidentified broad feature at $6525 \AA(F W H M \sim 5 \AA, \mathrm{EQW}=173 \mathrm{~m} \AA)$ is detected in the Tc 1 spectrum (Fig. 2). Figure 2 shows that the $6525 \AA$ band is real because it is not detected in the spectrum of the comparison star HR 6334 taken with the same UVES setup and at the same time.

\section{Fullerenes - DIB connection}

Our finding of an unusually strong $4428 \AA$ DIB towards Tc 1 (see left panel of Fig. 1) necessarily prompts the idea that the $4428 \AA$ DIB carrier may be related with fullerenes or fullerene-based molecules (Iglesias-Groth 2007). Remarkably, photo-absorption theoretical models of several large fullerenes such as $\mathrm{C}_{80}, \mathrm{C}_{240}$, 
$\mathrm{C}_{320}$, and $\mathrm{C}_{540}$ predict their strongest transitions very close in wavelength $( \pm 10 \AA)$ to this $4428 \AA$ DIB (Iglesias-Groth 2007$)^{5}$. The theoretical spectra of several multishell fullerenes (buckyonions such as $\mathrm{C}_{60} @ \mathrm{C}_{240}$ and $\mathrm{C}_{60} @ \mathrm{C}_{240} @ \mathrm{C}_{540}$ ) reported by Iglesias-Groth (2007) also display a strong $4428 \AA$ band. In this context, the broad $4428 \AA$ band may be well explained by the superposition of the transitions of fullerenes bigger than $\mathrm{C}_{60}$ and buckyonions. This would be consistent with the recent exhaustive study of the $4428 \AA$ DIB by van Loon et al. (2012), which suggests the carrier to be a large, compact, and neutral molecule that is relatively resistant to impacting energetic photons or particles.

Another interesting feature is that our Tc 1 spectra also lack the unidentified $4000 \AA$ band (see Fig. A. 1 in the Appendix) that is detected in the RCB star DY Cen (García-Hernández et al. 2012b). García-Hernández et al. (2012b) suggested that the midIR features at $\sim 7.0,8.5,17.4$, and $18.8 \mu \mathrm{m}$ and the unidentified $4000 \AA$ band in DY Cen are likely due to proto-fullerenes (PFs) or fullerene precursors rather than to $\mathrm{C}_{60}$. Interestingly, DY Cen displays a "normal" $4428 \AA$ DIB, supporting the claim that fullerenes and fullerene-based molecules such as buckyonions are not especially overabundant toward DY Cen. Thus, the unusually strong $4428 \AA$ DIB and the lack of the unidentified $4000 \AA$ band in Tc 1 may indicate an efficient conversion of PFs to fullerenes and fullerene-based molecules in its circumstellar envelope (see e.g., Duley \& Hu 2012).

Furthermore, the apparent lack of the strongest electronic transitions of the $\mathrm{C}_{60}$ molecule in Tc 1 may indicate that we are not seeing emission from isolated, free $\mathrm{C}_{60}$ molecules, which would explain the variable properties of the mid-IR $\mathrm{C}_{60}$ spectral features observed in fullerene PNe (Bernard-Salas et al. 2012; García-Hernández et al. 2012a). At present, the HAC's photochemical processing is the most likely $\mathrm{C}_{60}$ formation route in the complex circumstellar envelopes of PNe (García-Hernández et al. 2012a; Bernard-Salas et al. 2012; Micelotta et al. 2012). Larger fullerenes may grow from pre-existing $\mathrm{C}_{60}$ molecules (Dunk et al. 2012) that may be supplied by the photochemical processing of HAC dust, opening the possibility of forming other fullerene-based molecules such as buckyonions and fullerene adducts. Indeed, fullerenes and PAHs may be mixed in the circumstellar envelopes of fullerene PNe (e.g., M 1-20) and fullerene/PAH adducts may form via Dies-Alder cycloaddition reactions (García-Hernández et al. 2013).

Fullerene clusters or fullerene-based molecules such as buckyonions, fullerene/PAH adducts may still be excited by stochastic heating (e.g., from UV photons) emitting through the same IR vibrational modes. Indeed, very recent laboratory work demonstrates that fullerene/PAH adducts - such as $\mathrm{C}_{60}$ /anthracene Diels-Alder adducts - display mid-IR features strikingly coincident with those from $\mathrm{C}_{60}$ and $\mathrm{C}_{70}$ (García-Hernández et al. 2013). Unfortunately, the synthesis of multishell fullerenes (buckyonions) in the laboratory is challenging because their insolubility does not permit us to extract and separate these species from the carbon soot in which they are present in small amounts (Cataldo, priv. comm.).
In summary, we propose that the $4428 \AA$ DIB (possibly also the $6309 \AA \mathrm{DIB}$ and the new $6525 \AA$ band) is probably related to fullerenes bigger than $\mathrm{C}_{60}$ (e.g., $\mathrm{C}_{80}, \mathrm{C}_{240}, \mathrm{C}_{320}$, and $\mathrm{C}_{540}$ ) and buckyonions (e.g., $\mathrm{C}_{60} @ \mathrm{C}_{240}, \mathrm{C}_{60} @ \mathrm{C}_{240} @ \mathrm{C}_{540}$ ) in the Tc 1 circumstellar environment. This possible fullerenes - DIB connection was previously suggested by Iglesias-Groth (2007) from theoretical considerations.

Acknowledgements. We acknowledge the referee Jacco van Loon for very useful comments that helped to improve the paper. We also acknowledge A. Manchado for his help during the early stage of this project and F. Cataldo for providing us with his fullerene laboratory data and expertise that helped to improve this Letter. This work is based on observations obtained with ESO/VLT under the program 087.D-0189(A). D.A.G.H. acknowledges support provided by the Spanish Ministry of Economy and Competitiveness under grant AYA-2011-27754.

\section{References}

Ajie, H., Alvarez, M. M., Anz, S. J., et al. 1990, J. Phys. Chem., 94, 8630 Ballester, P., Modigliani, A., Boitquin, O., et al. 2000, Messenger, 101, 31 Bernard-Salas, J., Cami, J., Peeters, E., et al. 2012, ApJ, 757, 41

Bettens, R. P. A., \& Herbst, E. 1996, ApJ, 468, 686

Cami, J., Bernard-Salas, J., Peeters, E., et al. 2010, Science, 329, 1180

Cataldo, F., \& Iglesias-Groth, S. 2009, MNRAS, 400, 291

Cox, N. L. J. 2011, in PAHs and the Universe: A Symposium to Celebrate the 25th Anniversary of the PAH Hypothesis, eds. C. Joblin, \& A. G. G. M. Tielens, EAS Pub. Ser., 46, 349

Duley, W. W., \& Hu, A. 2012, ApJ, 745, L11

Dunk, P. W., Kaiser, N. K., Hendrickson, C. L., et al. 2012, Nature Commun., 3, 855

Evans, A., van Loon, J. Th., Woodward, C. E., et al. 2012, MNRAS, 421, L92

Foing, B. H., \& Ehrenfreund, P. 1994, Nature, 369, 296

García-Hernández, D. A., Manchado, A., García-Lario, P., et al. 2010, ApJ, 724, L39

García-Hernández, D. A., Iglesias-Groth, S., Acosta-Pulido, J. A., et al. 2011a, ApJ, 737, L30

García-Hernández, D. A., Rao, N. K., \& Lambert, D. L. 2011b, ApJ, 729, 126

García-Hernández, D. A., Rao, N. K., \& Lambert, D. L. 2011c, ApJ, 739, 37

García-Hernández, D. A., Villaver, E., García-Lario, P., et al. 2012a, ApJ, 760, 107

García-Hernández, D. A., Rao, N. K., \& Lambert, D. L. 2012b, ApJ, 759, L21 García-Hernández, D. A., Cataldo, F., \& Manchado, A. 2013, MNRAS, submitted

Geballe, T. R., Najarro, F., Figer, D. F., et al. 2011, Nature, 479, 200

Heger, M. L. 1922, Lick Obs. Bull. 10, 146

Herbig, G. H. 1995, ARA\&A, 33, 19

Herbig, G. H. 2000, ApJ, 542, 334

Hobbs, L. M., York, D. G., Snow, T. P., et al. 2008, ApJ, 680, 1256

Iglesias-Groth, S. 2004, ApJ, 608, L37

Iglesias-Groth, S. 2007, ApJ, 661, L167

Kroto, H. W., \& Jura, M. 1992, A\&A, 263, 275

Luna, R., Cox, N. L. J., Satorre, M. A., et al. 2008, A\&A, 480, 133

Maier, J. P., Walker, G. A. H., Bohlender, D. A., et al. 2011, ApJ, 726, 41

Micelotta, E. R., Jones, A. P., Cami, J., et al. 2012, ApJ, 761, 35

Pottasch, S. R., Surendiranath, R., \& Bernard-Salas, J. 2011, A\&A, 531, A23

Sassara, A., Zerza, G., Chergui, M., et al. 2001, ApJ, 135, 263

Sellgren, K., Werner, M. W., Ingalls, J. G., et al. 2010, ApJ, 722, L54

Snow, T. P., \& McCall, B. J. 2006, ARA\&A, 44, 367

Snow, T. P., Zukowski, D., \& Massey, P. 2002, ApJ, 578, 877

Salama, F., Galazutdinov, G. A., Krelowski, J., et al. 1999, ApJ, 526, 265

van Loon, J. Th., Bailey, M., Tatton, B. L., et al. 2013, A\&A, DOI:10.1051/0004$6361 / 201220210$

Wang, W., \& Liu, X.-W. 2007, MNRAS, 381, 669

Wegner, W. 2003, AN, 324, 219

Williams, R., Jenkins, E. B., Baldwin, J. A., et al. 2008, ApJ, 677, 1100

Page 5 is available in the electronic edition of the journal at http://wwW . aanda. org

\footnotetext{
5 The same models do not predict a $4428 \AA$ band for the $\mathrm{C}_{60}$ fullerene, in agreement with fullerene laboratory spectroscopy (e.g., Sassara et al. 2001).
} 
Appendix A: Figures A.1 and A.2 and Table A.1
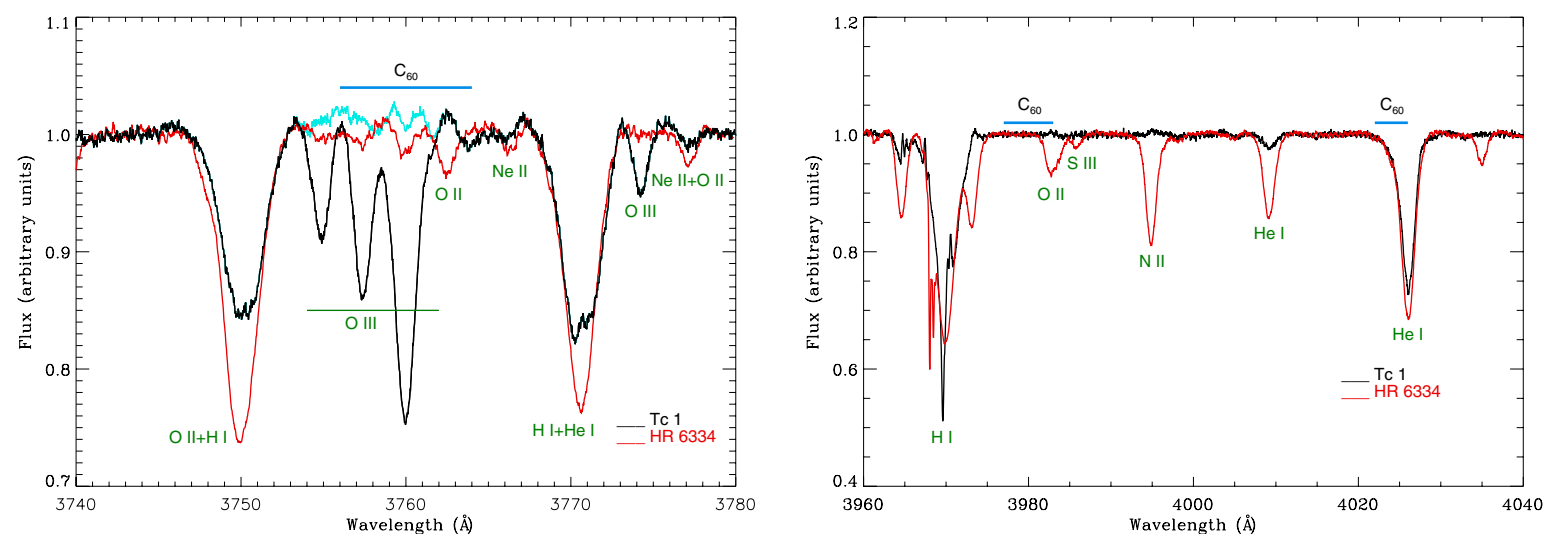

Fig. A.1. Velocity-corrected spectra of Tc 1 (in black) and HR 6334 (in red) around $3760 \AA$ (left panel) and $4000 \AA$ (right panel) where the atomic line identifications are indicated (in green). The expected positions (and FWHMs) of the $\mathrm{C}_{60}$ features are marked on top of the spectra. There is no evidence (additional absorption) in Tc 1 for the neutral $\mathrm{C}_{60}$ features at 3760, 3980, and $4024 \AA$. The residual spectrum (in cyan) obtained by subtracting the oxygen lines around $3760 \AA$ is also shown.

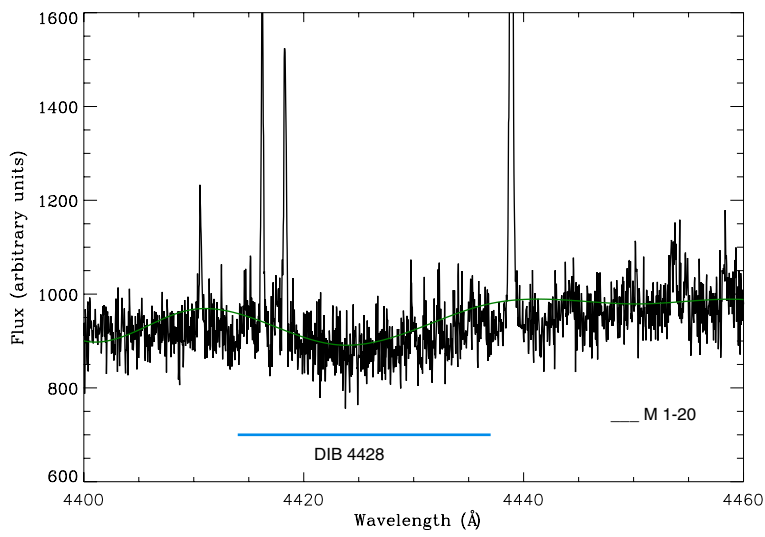

Fig. A.2. Spectrum of M 1-20 (in black) around the broad $4428 \AA$ DIB. A heavily smoothed and emission line corrected spectrum is also overplotted (in green).

Table A.1. Diffuse interstellar bands in fullerene $\mathrm{PNe}^{a}$.

\begin{tabular}{lccccccccccc}
\hline \hline $\begin{array}{l}\mathrm{Tc} 1 \\
\lambda_{\mathrm{c}} \\
(\AA)\end{array}$ & $\begin{array}{c}F W H M \\
(\AA)\end{array}$ & $\begin{array}{c}\mathrm{EQW} \\
(\mathrm{m} \AA)\end{array}$ & $S / N$ & $\begin{array}{c}\mathrm{EQW} / E_{B-V} \\
(\AA / \mathrm{mag})\end{array}$ & $\begin{array}{c}\mathrm{M} 1-20 \\
\lambda_{\mathrm{c}} \\
(\AA)\end{array}$ & $\begin{array}{c}F W H M \\
(\AA)\end{array}$ & $\begin{array}{c}\mathrm{EQW} \\
(\mathrm{m} \AA)\end{array}$ & $\begin{array}{c}S / N \\
\mathrm{EQW} / E_{B-V} \\
(\AA / \mathrm{mag})\end{array}$ & $\begin{array}{c}\text { Hobbs et al. } \\
\mathrm{EQW} / E_{B-V} \\
(\AA / \mathrm{mag})\end{array}$ & $\begin{array}{c}\text { Luna et al. } \\
\mathrm{EQW} / E_{B-V} \\
(\AA / \mathrm{mag})\end{array}$ \\
\hline $4428.10^{b}$ & 19.35 & 860 & 329 & 3.74 & $4426.56^{b}$ & $19.94^{c}$ & $2579^{c}$ & $20^{d}$ & 3.22 & 1.10 & $\ldots$ \\
5780.40 & 2.06 & 105 & 416 & 0.46 & 5780.66 & 2.17 & 359 & 32 & 0.45 & 0.23 & 0.46 \\
5796.88 & 0.99 & 42 & 357 & 0.18 & 5797.31 & 1.14 & 155 & 53 & 0.19 & 0.18 & 0.17 \\
5849.60 & 1.35 & 11 & 346 & 0.047 & 5850.02 & 1.23 & 70 & 37 & 0.087 & 0.086 & 0.061 \\
6195.85 & 1.17 & 13 & 549 & 0.056 & 6196.18 & 0.95 & 36 & 68 & 0.045 & 0.034 & 0.053 \\
6269.77 & 1.93 & 9 & 548 & 0.037 & 6270.19 & 2.48 & 130 & 66 & 0.16 & 0.069 & $\ldots$ \\
6283.93 & 4.91 & 316 & 579 & 1.38 & 6283.77 & 5.25 & 706 & 61 & 0.88 & 0.41 & 0.90 \\
6308.90 & 2.98 & 42 & 530 & 0.18 & $\ldots$ & $\ldots$ & $\ldots$ & $\ldots$ & $\ldots$ & 0.049 & $\ldots$ \\
6379.08 & 1.39 & 12 & 513 & 0.050 & 6379.56 & 1.30 & 93 & 56 & 0.12 & 0.085 & 0.088 \\
6525.15 & 4.79 & 173 & 522 & 0.75 & $\ldots$ & $\ldots$ & $\ldots$ & $\ldots$ & $\ldots$ & $\ldots$ & $\ldots$ \\
6613.50 & 1.42 & 36 & 412 & 0.16 & 6613.78 & 1.23 & 167 & 77 & 0.21 & 0.149 & 0.21 \\
\hline
\end{tabular}

Notes. ${ }^{(a)}$ The $3-\sigma$ errors in the EQWs scale like $\sim 3 \times \mathrm{FWHM} /(\mathrm{S} / \mathrm{N})$ while we estimate that the FHWMs in Tc 1 are precise to the $0.03 \AA$ level (less for M 1-20). ${ }^{(b)}$ The characteristics of this DIB are estimated by adopting a Lorentzian profile (see e.g., Snow et al. 1992). ${ }^{(c)}$ Best estimates found by clipping out the narrow emission lines and smoothing the spectrum with boxcar 15 . The error in the quoted EQW is estimated to be $\sim 786 \mathrm{~m} \AA$. (d) $\mathrm{S} / \mathrm{N}$ in the original spectrum. 